Leslie WD, Aubry-Rozier B, Lamy O, Hans D. TBS (Trabecular Bone Score) and Diabetes-Related Fracture Risk. J Clin EndocrinolMetab.2013;98:6029. -Kim JH, Choi HJ, Ku EJ, Kim KM, Kim SW, Cho NH, et al. Trabecular Bone Score as an Indicator for Skeletal Deterioration in Diabetes. J Clin EndocrinolMetab.2015;100:475-82.

Disclosure of Interests: None declared DOI: 10.1136/annrheumdis-2019-eular.7199

\section{FRI0472 \\ COMPARISON OF THE THERAPEUTIC EFFICACY OF DAILY VERSUS WEEKLY DOSE OF TERIPARATIDE FOR PREVENTING BONE FRACTURE IN PATIENTS WITH OSTEOPOROSIS - A SINGLE CENTRE, PROSPECTIVE OPEN-LABEL STUDY}

Takafumi Hagiwara, Kazuya Kamada, Noriyuki Namura. Takarazuka City Hospital, Rheumatology, Takarazuka, Japan

Background: In Japan, teriparatide $(20 \mu \mathrm{g})$ and teriparatide acetate $(56.5$ $\mu \mathrm{g})$ have been approved for treatment of osteoporosis. Teriparatide is a recombinant human parathyroid hormone (PTH) administered as daily subcutaneous injections, whereas teriparatide acetate is a biologically active fragment containing the N-terminal 34 amino acids of human PTH administered as weekly subcutaneous injections. To the best of our knowledge, a study comparing the therapeutic effects of these two teriparatide preparations does not exist.

Objectives: We aimed to compare the therapeutic effects of the two teriparatide preparations that varied in dose interval.

Methods: We prospectively enrolled 55 osteoporotic patients with vertebral fracture who needed bone-strengthening therapy in a single-centre cohort. The patients randomly selected either daily injection of teriparatide for 2 years or weekly injection of teriparatide acetate for 72 weeks. The primary endpoint was comparison of the new fracture onset ratio at 18 months, based on the approval for use of teriparatide and teriparatide acetate for up to 2 years and 72 weeks, respectively at the time of the trial. However, since May 2017, teriparatide acetate use for osteoporosis treatment has been approved for up to 24 months in Japan. We evaluated their background data (age, sex, bisphosphonate use, basal disease, glucocorticoid use, vertebral and femoral bone marrow density (BMD), number of thoracic and lumbar vertebral fractures, levels of tartrate-resistant acid phosphatase 5b (TRACP-5b), total procollagen type 1 amino-terminal propeptide (total P1NP), 1,25(OH)2-vitamin D3 $1,25(\mathrm{OH})$ 2D3), total serum calcium corrected for albumin level (cCa), inorganic phosphorus(IP), homocysteine, whole PTH and estimated glomerular filtration rate, ankle-brachial index(ABI), and brachial-ankle pulse wave velocity (baPWV)). IP levels were observed until month 1 , while levels of TRACP-5b, total P1NP, cCa, 1,25(OH)2D3, BMD, number of fractured vertebrae, $\mathrm{ABI}$, and baPWV were observed until month 18 .

Results: The background factors were not significantly different between the groups. The onset rates of new vertebral fractures until 18 months were $50 \%$ and $52 \%$ for teriparatide and teriparatide acetate, respectively. However, we did not observe the onset of new non-vertebral fracture in the two groups for 18 months. Survival rates in new bone fracture onset (teriparatide vs teriparatide acetate) were $71.4 \%$ vs. $60.0 \%$ at month 6 , $57.1 \%$ vs. $52.2 \%$ at month 12 , and $46.4 \%$ vs. $52.2 \%$ at month 18 . The survival curve showed no significant difference in new bone fracture onset between the groups using the log rank test $(p=.976)$ with a hazard ratio (teriparatide vs. teriparatide acetate) of 0.867 (95\% confidential interval; 0.291-2.582) $(p=.797) \quad$ (see Fig.1). No significant differences were seen in the continuation rates for 18 months for teriparatide (93.3\%) and teriparatide acetate $(96.0 \%)(p=.669)$. Statistically significant differences in $\% \Delta$ vertebral BMD at month 6 and baseline $(p<.01)$; TRACP-5b at months 12 and $18 \quad(p<.05)$; total P1NP at months $1,6,12$, and $18 \quad(p<.01)$; and $\mathrm{cCa}$ at month $6(\mathrm{p}<.01)$ were seen between the groups. Moreover, levels of $1,25(\mathrm{OH}) 2 \mathrm{D} 3$ at months 6,12 , and 18 were lower with teriparatide acetate than those of teriparatide $(p<.01)$.

Conclusion: The protective efficacy of the teriparatide preparations to prevent bone fracture showed no difference. In osteoporosis treatment with vertebral fracture, teriparatide preparation daily or weekly may be selected based on the patient's choice.

[UMIN000024503]

\section{REFERENCES:}

[1] J Clin Endocrinol Metab. 2012 Sep;97(9):3097-106.

[2] Arthritis Rheum. $2009 \mathrm{Nov} ; 60(11): 3346-55$.
DOI: 10.1136/annrheumdis-2019-eular.1316

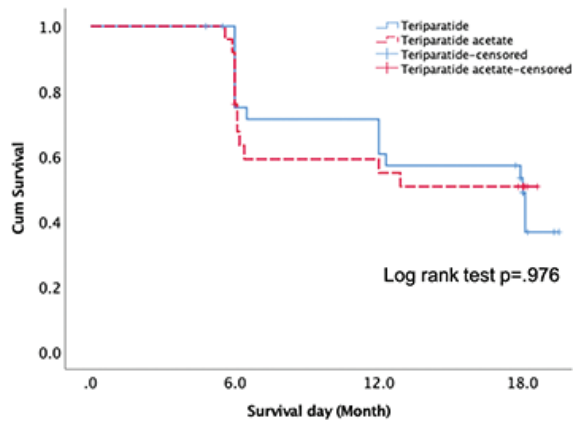

Figure. 1 Survival curve in new bone fracture onset of teriparatide and teriparatide acetate.

\section{FRI0473 \\ ASSESSING THE CLINICAL RELEVANCE OF BONE DENSITOMETRY SCREENING IN PATIENTS UNDER 40 YEARS OF AGE: INDICATIONS AND RESULTS}

Laura Chadwick, Deepti Challa, Sankalp Tripathi, Kiran Kumar Putchakayala. Mid Cheshire Hospitals NHS Trust, Crewe, United Kingdom

Background: Guidelines for the diagnosis and treatment of osteoporosis in patients under the age of 40 years are lacking. The International Osteoporosis Foundation (IOF) working group has recommended dualenergy X-ray absorption (DXA) screening should only be performed in this age group if patients have an associated chronic disease such as inflammatory bowel disease (IBD) in conjunction with a vertebral fracture or multiple fractures elsewhere. ${ }^{1}$ For these patients, a T-score of $<-2.5$ can be used to diagnosis low BMD but the term osteoporosis may not be appropriate and there is minimal evidence supporting the use of bisphosphonates (BPs).

Objectives: In patients under 40 years of age undergoing DXA screening, to determine (1) the indication and (2) the DXA results (age adjusted Z score and standard $\mathrm{T}$ score), in order to conclude whether DXA screening in this group was appropriate and relevant for making meaningful treatment decisions.

Methods: Clinical details for all DXA scans requested for patients less than 40 years of age were obtained from the Mid Cheshire Hospital NHS Trust radiology department for a 6 month period from July to December 2018 inclusive. Descriptive data was produced regarding basic patient demographics, DXA indication and DXA results in the form of Zand T-scores at the neck of femur (NOF) and spine.

Results: In total, 35 patients under the age of 40 years underwent DXA screening in the defined time period. The mean age of patients was 30.5 years (s.d. 6.0) and 24 patients (69\%) were female. The youngest patients were 19 years. Regarding indications, only two patients $(6 \%)$ were appropriate for DXA screening according to the IOF suggested guidelines, both of whom had vertebral fractures. Twenty seven patients (77\%) had an established diagnosis of a disease known to cause OP but no history of fracture. Regarding results, at the NOF and spine respectively mean Z-scores were -0.77 (s.d. 1.19) and -0.96 (s.d. 0.97), and mean T-scores were -0.88 (s.d. 1.19) and -0.945 (0.93). Only three patients $(9 \%)$ had Z- or T-score of $<-2.5$; two patients who had a history of thoracic vertebral fracture, and a patient with high steroid use for IBD but no fracture history.

Conclusion: The majority of patients aged less than 40 years referred for DXA screening are being referred due to associated diagnoses with no history of fracture, not in keeping with IOF recommendations. Of the 33 patients with no history of fracture, only one patient $(3 \%)$ had a Tscore consistent with low BMD. There is little evidence to support bisphosphonate use in this group. We therefore conclude that DXA screening in patients less than 40 years should be reserved for those with a history of fracture.

\section{REFERENCES:}

[1] Ferrari, S. et al. (2012). Osteoporosis in young adults: pathophysiology, diagnosis and management. Osteoporosis Int. 23:2735-2748

Disclosure of Interests: None declared DOI: 10.1136/annrheumdis-2019-eular.3304 